\title{
GLOBAL SOLUTIONS OF MAXWELL'S EQUATIONS IN AN ELECTROMAGNETIC FIELD WITH THE TEMPERATURE-DEPENDENT ELECTRICAL CONDUCTIVITY
}

\author{
By \\ Hong-Ming Yin
}

IMA Preprint Series \# 899

December 1991 


\title{
Global Solutions of Maxwell's Equations in an Electromagnetic
}

\section{Field with the Temperature-Dependent Electrical Conductivity ${ }^{1}$}

\author{
Hong-Ming Yin \\ Department of Mathematics \\ University of Toronto \\ Toronto, Ontario M5S 1A1, Canada
}

\begin{abstract}
This paper deals with Maxwell's equations in a quasi-stationary electromagnetic field subject to the effects of the temperature field. This model is encountered in the penetration of a magnetic field in substances where the electrical conductivity depends on the temperature. Similar phenomena also occur in some industrial problems such as the thermister. Taking the effect of the Joule heat into the consideration, we obtain a strongly coupled nonlinear system. The global solvability is established for this system.
\end{abstract}

Key Words and Phrases: Maxwell's Equations, coupled nonlinear system, global solvability.

AMS(MOS)Subject Classications: 35Q20, 35K55, 78A97.

\footnotetext{
${ }^{1}$ This work is supported by NSERC of Canada
} 


\section{Introduction}

In a quasi-stationary electromagnetic field the magnetic induction $\vec{B}$, the magnetic field $\vec{H}$ and the electric field $\vec{E}$ satisfy the approximate Maxwell equations (cf. Landau and Lifshtz [4], page 186-187):

$$
\begin{aligned}
& \operatorname{div} \vec{B}=0, \\
& \operatorname{curl} \vec{H}=\frac{4 \pi \sigma}{c} \vec{E}, \\
& \operatorname{curl} \vec{E}=-\frac{1}{c} \frac{\partial \vec{B}}{\partial t},
\end{aligned}
$$

where $\sigma$ is the electrical conductivity and $c$ is a physical constant. With nonferromagnetic bodies, if the strength of magnetic field is weak, the magnetic induction is proportional to the strength of the magnetic field (cf. [4], page 120), that is

$$
\vec{B}=\mu \vec{H},
$$

where $\mu$ is the magnetic permeability.

In the study of the penetration of a magnetic field in a medium the electrical resistance strongly depends on the temperature. On the other hand, the electric current $\vec{J}$ satisfies Ohm's law (cf. [4], [7]):

$$
\vec{J}=\sigma(u) \vec{E} .
$$

It follows that the heat source produced by the Joule heat is

$$
\begin{aligned}
& \vec{J} \cdot \vec{E}=\sigma(u) \vec{E} \cdot \vec{E} \\
& =\frac{c}{4 \pi \sigma(u)}|\nabla \times \vec{H}|^{2},
\end{aligned}
$$


where the equation (1.2) has been used in the above derivation.

With the assumption of the constant permeability, we have, after normalizing the constants, the following form of Maxwell's equations and the heat equation:

$$
\begin{aligned}
& \operatorname{div} \vec{H}=0, \\
& \frac{\partial \vec{H}}{\partial t}=-\nabla \times[k(u) \nabla \times \vec{H}], \\
& u_{t}-\Delta u=k(u)|\nabla \times \vec{H}|^{2},
\end{aligned}
$$

where $k(u)=\frac{1}{\sigma(u)}$ is so-called the resistivity of the material.

Obviously, the equation (1.6) is true as a result of (1.7) if the initial state satisfies this equation. It is our objective in the present paper to study the equations (1.7)(1.8) subject to appropriate initial and boundary conditions.

It is easy to see that if we take $\vec{H}=\{w, 0,0\}$, the system (1.6)-(1.8) is reduced into a system which is similar to the thermister problem in an electric field (steadystate case):

$$
\begin{aligned}
& \nabla[k(u) \nabla w]=0, \\
& -\Delta u=k(u)|\nabla w|^{2} .
\end{aligned}
$$

This problem has been studied by many authors (cf. [1], [2], [6], for example, and the references listed therein). In these previous studies, the maximum principle plays an essential role in the proof of the existence. However, our system is much more complicated. The maximum principle can no longer be applied for the magnetic field $\vec{H}(x, t)$. We shall seek the weak solvability. With the help of Simon's compactness results (cf. [5]), we are able to establish the global existence.

In Section 2, we will state the problem in a weak form and define the weak solution. In Section 3, we construct an approximate problem and show that the 
approximate problem admits a unique classical solution; then the compactness argument is used to prove the existence of a weak solution.

\section{Weak Formulation}

For reader's convenience, we list some notions below. Let $B$ be a Banach space and - $1 \leq p<\infty$,

$$
L^{p}(0, T ; B)=\left\{f \mid f:[0, T] \rightarrow B \text { with the norm }\|f\|_{L^{p}(0, T ; B)}<\infty,\right\}
$$

where

$$
\|f\|_{L^{p}(0, T ; B)}=\left[\int_{0}^{T}\|f\|_{B}^{p} d t\right]^{\frac{1}{p}} .
$$

The spaces $W^{m, k}(\Omega), H_{0}^{1}(\Omega)$, etc. are the common Banach spaces with the usual norms, and $B^{m}$ denotes the product space $B \times B \cdots \times B$. Let $\Omega$ be a bounded domain in $R^{3}$ and $Q_{T}=\Omega \times(0, T]$. The initial and boundary conditions are given by

$$
\begin{array}{ll}
\vec{H}(x, t)=\vec{F}(x, t), & (x, t) \in S_{T}=\partial \Omega \times(0, T] ; \\
u(x, t)=g(x, t), \quad(x, t) \in S_{T} ; \\
\vec{H}(x, 0)=\vec{H}_{0}(x), \quad x \in \Omega ; \\
u(x, 0)=u_{0}(x), \quad x \in \Omega .
\end{array}
$$

Without loss of generality, we may assume that $g(x, t)=0$. A weak solution of the problem (1.7)-(1.8), (2.1)-(2.4) is defined as follows:

Definition: A pair of functions $(\vec{H}(x, t), u(x, t))$ is said to be a weak solution of the problem (1.7)-(1.8), (2.1)-(2.4) if $\vec{H}(x, t)-\vec{F}(x, t) \in L^{2}\left(0, T ; H_{0}^{1}(\Omega)^{3}\right)$ with 
$\operatorname{div} \cdot \vec{H}(x, t)=0$ a.e. in $Q_{T}$, and $u(x, t) \in L^{\infty}\left(0, T ; L^{1}(\Omega)\right) \cap L^{1}\left(0, T ; W_{0}^{1,1}(\Omega)\right)$, which satisfy the following integral identities:

$$
\begin{aligned}
& \iint_{Q_{T}}\left[-\vec{H} \cdot \vec{\psi}_{t}+k(u) \nabla \times \vec{H} \cdot \nabla \times \vec{\psi}\right] d x d t-\int_{\Omega} \vec{H}_{0}(x) \cdot \vec{\psi}(x, 0) d x=0 \\
& \iint_{\Omega} u\left[\phi_{t}+\Delta \phi\right] d x d t=-\iint_{\Omega} k(u)|\nabla \times \vec{H}|^{2} \phi d x d t
\end{aligned}
$$

for any $\vec{\psi}(x, t) \in H^{1}\left(0, T ; H_{0}^{1}(\Omega)^{3}\right)$ with $\vec{\psi}(x, T)=0$ and for any $\phi(x, t) \in C_{0}^{\infty}\left(Q_{T}\right)$. Moreover, the limit of $u(x, t)$ as $t$ tends to 0 is $u_{0}(x)$ in the sense of Lebesgue measure.

The following lemma plays a fundamental role in proving the existence. The reader can consult J. Simon's paper [5]( Corollary 4, on page 85) for its proof.

Lemma 2.1: Let $X, B$ and $Y$ be Banach spaces with $X \subset B \subset Y$. Moerover, the imbedding operator from $X \rightarrow B$ is compact. Let $F$ be a set of functions from $[0, T] \rightarrow X$. If $F$ is bounded in $L^{p}(0, T ; X)$, where $1 \leq p<\infty$, and

$$
\frac{\partial F}{\partial t}=\left\{\frac{\partial f}{\partial t}: f \in F\right\} \text { is bounded in } L^{1}(0, T ; Y) \text {. }
$$

Then $F$ is relatively compact in $L^{p}(0, T ; B)$.

Throughout this paper, we assume:

$\mathbf{H}(1)$ : The function $k(u)$ is continuous in $R^{1}$, and there exist two constants $k_{1}$ and $k_{2}$ such that

$$
0<k_{1} \leq k(u) \leq k_{2} .
$$

H(2): The function $\vec{F}(x, t)$ can be extended into $Q_{T}$ such that the extended function $\vec{F}(x, t) \in W_{p}^{2,1}\left(Q_{T}\right)^{3}$, the initial data $\vec{H}_{0}(x) \in W_{p}^{2}(\Omega)^{3}$ with $\operatorname{div} \vec{H}_{0}(x)=0$ and $p>\frac{5}{2}$ and $u_{0}(x) \in L^{2}(\Omega)$. Moreover, the following consistency condition is satisfied:

$$
\vec{F}(x, 0)=\vec{H}_{0}(x), \quad x \in \partial \Omega .
$$


Our main result is

Theorem: Under the assumptions $\mathrm{H}(1)-\mathrm{H}(2)$, the problem (1.6)-(1.8), (2.1)-(2.4) admits a weak solution globally in time.

\section{Proof}

We start with the following approximate problem:

$$
\begin{aligned}
& \vec{H}_{t}=-\nabla \times[k(u) \nabla \times \vec{H}], \quad(x, t) \in Q_{T}=\Omega \times(0, T], \\
& u_{t}=\Delta u+\frac{k(u)|\nabla \times \vec{H}|^{2}}{1+\varepsilon k(u)|\nabla \times \vec{H}|^{2}}, \quad(x, t) \in Q_{T},
\end{aligned}
$$

subject to the initial and boundary conditions $(2.1)-(2.4)$, where $\vec{H}_{0}(x)$ and $u_{0}(x)$ are replaced by the smooth approximation functions $\vec{H}_{0 \varepsilon}(x)$ and $u_{0 \varepsilon}(x)$ such that $\operatorname{div} \vec{H}_{0 \varepsilon}=0$ and the consistency conditions hold up to the second order.

Lemma 3.1: The approximate problem admits a unique classical solution:

$$
\vec{H}_{\varepsilon}(x, t) \in C^{2,1}\left(\bar{Q}_{T}\right)^{3} ; u_{\varepsilon}(x, t) \in C^{2,1}\left(\bar{Q}_{T}\right) .
$$

Moreover, the following estimates hold:

$$
\begin{aligned}
& \operatorname{div} \vec{H}_{\varepsilon}(x, t)=0, \quad \text { in } Q_{T}, \\
& \sup _{0 \leq t \leq T} \int_{\Omega}\left|\vec{H}_{\varepsilon}\right|^{2} d x+\iint_{Q_{T}} k(u)\left|\nabla \times \vec{H}_{\varepsilon}\right|^{2} d x d t \leq C_{1}, \\
& \sup _{0 \leq t \leq T} \int_{\Omega}|u| d x+\iint_{Q_{T}}|\nabla u| d x d t \leq C_{2},
\end{aligned}
$$

where the constants $C_{1}$ and $C_{2}$ depends only on the known data, but not on $\varepsilon$. Proof: The existence can be shown easily by the Schauder fixed point theorem since the nonlinear term in (3.2) is bounded. The proof of uniqueness is also standard, 
and so it only remains to derive the estimates (3.3)-(3.4). First of all, we take the divergence in the equation (3.1) to see

$$
\left(\operatorname{div} \vec{H}_{\varepsilon}\right)_{t}=0
$$

which implies

$$
\operatorname{div} \vec{H}_{\varepsilon}(x, t)=\operatorname{div} \vec{H}_{0 \varepsilon}(x)=0 .
$$

Next, we note that for $u(x) \in H_{0}^{1}(\Omega)$,

$$
\int_{\Omega}(\nabla \times \vec{u}) \cdot \vec{v} d x=\int_{\Omega} \vec{u} \cdot(\nabla \times \vec{v}) d x .
$$

If we take the inner product to (3.1) with $\vec{H}_{\varepsilon}-\vec{F}$, we have

$$
\begin{aligned}
& \frac{1}{2} \int_{\Omega}\left[\left|\vec{H}_{\varepsilon}-\vec{F}\right|^{2}-\left|\vec{H}_{0 \varepsilon}(x)-\vec{F}(x, 0)\right|^{2}\right] d x+\iint_{Q_{T}}\left(\vec{H}_{\varepsilon}-\vec{F}\right) \cdot \vec{F} d x d t \\
& =-\iint_{Q_{T}} k(u)\left|\nabla \times \vec{H}_{\varepsilon}\right|^{2} d x d t+\iint_{Q_{T}} k(u)\left(\nabla \times \vec{H}_{\varepsilon}\right) \cdot(\nabla \times \vec{F}) d x d t .
\end{aligned}
$$

By Cauchy-Schwartz's inequality,

$$
\iint_{Q_{T}}\left(\vec{H}_{\varepsilon}-\vec{F}\right) \cdot \vec{F}_{t} d x d t \leq \delta \iint_{Q_{T}}\left|\vec{H}_{\varepsilon}-\vec{F}\right|^{2} d x d t+C(\delta) \iint_{Q_{T}}\left|\vec{F}_{t}\right|^{2} d x d t .
$$

Now taking $\delta$ sufficiently small and using Gronwall's inequality, we have the desired estimate (3.3).

To prove the estimate (3.4), we need the following lemma:

Lemma 3.2: Assume that $u(x, t)$ is a distribution solution of the following problem: $u(x, t) \in L^{1}\left(0, T ; W_{0}^{1,1}(\Omega)\right)$,

$$
\begin{aligned}
& u_{t}-\triangle u=f(x, t), \quad(x, t) \in Q_{T}, \\
& u(x, 0)=u_{0}(x), \quad x \in \Omega,
\end{aligned}
$$


where $f(x, t) \in L^{1}\left(Q_{T}\right)$. Then the following estimate holds

$$
\sup _{0 \leq t \leq T} \int_{\Omega}|u| d x+\iint_{Q_{T}}|D u| d x d t \leq C .
$$

This result can be shown by using Green's representation. For completeness, we sketch the proof. We may assume that the initial value and the inhomogenous term are smooth. Then Green's representation gives

$$
u(x, t)=\int_{\Omega} G(x, y ; t, 0) u_{0}(y) d y+\iint_{Q_{T}} G f d y d \tau .
$$

Observe that

$$
\int_{\Omega} G d x \leq C
$$

and also that

$$
\int_{\Omega}|D G(x, y, t, \tau)| d x \leq \frac{C}{\sqrt{t-\tau}}, \quad t>\tau
$$

it follows that

$$
\int_{\Omega}|u| d x \leq C\left[\left\|u_{0}\right\|_{L^{1}(\Omega)}+\|f\|_{L^{1}\left(Q_{T}\right)}\right] .
$$

The second estimate can be proved in a similar manner.

Now we are ready to prove the main theorem.

The proof of the Theorem: First of all, we have the following identity:

$$
\int_{\Omega} \sum_{i=1}^{3}\left(u_{i x}^{2}+u_{i y}^{2}+u_{i z}^{2}\right) d x=\int_{\Omega}(d i v \cdot \vec{u})^{2} d x+\int_{\Omega}|\nabla \times \vec{u}|^{2} d x
$$

for any $\vec{u} \in H_{0}^{1}(\Omega)^{3}$. This can be proved by direct calculations.

By Lemma 3.1 and the above property we know

$$
\left\{\vec{H}_{\varepsilon}\right\} \in L^{2}\left(0, T ; H_{0}^{1}(\Omega)^{3}\right) .
$$


From the equation (3.1), we also have

$$
\frac{\partial \vec{H}_{\varepsilon}}{\partial t} \in L^{2}\left(0, T ; H^{-1}(\Omega)^{3}\right) \text {. }
$$

Lemma 2.1 implies that there exists a subsequence of $\left\{\vec{H}_{\varepsilon}(x, t)\right\}$, still denoted by itself, which converges to a function almost everywhere in $Q_{T}$. We denote the limit function by $\vec{H}(x, t)$. Moreover, the weak compactness of $L^{2}\left(Q_{T}\right)$ implies that

$$
\nabla \times \vec{H}_{\varepsilon}(x, t) \rightarrow \nabla \times \vec{H}(x, t)
$$

in $L^{2}\left(Q_{T}\right)^{3}$ weakly.

Now $\left\{u_{\varepsilon}(x, t)\right\} \in L^{1}\left(0, T ; W_{0}^{1,1}(\Omega)\right)$, hence, $\nabla u \in L^{1}\left(0, T ; L^{1}(\Omega)^{3}\right)$, and so

$$
\triangle u \in L^{1}\left(0, T ;\left(W_{0}^{1, \infty}(\Omega)\right)^{\prime}\right),
$$

where $\left(W_{0}^{1, \infty}(\Omega)\right)^{\prime}$ denotes the conjugate space of $W_{0}^{1, \infty}(\Omega)$. As $L^{1}(\Omega) \subset\left(W_{0}^{1, \infty}(\Omega)\right)^{\prime}$, it follows by the equation (3.2) that

$$
\left\{\frac{\partial u_{\varepsilon}}{\partial t}\right\} \in L^{1}\left(0, T ;\left(W^{1, \infty}(\Omega)\right)^{\prime}\right) .
$$

Moreover, since the imbedding operator from $W_{0}^{1,1}(\Omega)$ into $B V(\Omega)$ is compact (cf.[3]), where $B V(\Omega)$ denotes the Banach space which is composed of all of functions with finite total variations. It follows by Lemma 2.1 that $\left\{u_{\varepsilon}(x, t)\right\}$ is relatively compact in $L^{1}\left(0, T ; B V(\Omega)\right.$ ). Consequently, there exists a subsequence of $\left\{u_{\varepsilon}\right\}$ (denoted by itself) which converges to a function denoted by $u(x, t)$ a.e. in $Q_{T}$.

Now we are ready to show that $\{\vec{H}(x, t), u(x, t)\}$ is a weak solution. The difficulty lies in the nonlinear term $\left|\nabla \times \vec{H}_{\varepsilon}\right|^{2}$ since we only know that $\nabla \times \vec{H}_{\varepsilon}$ converges weakly. However, this difficulty can be overcomed by using the first equation (2.5). 
The integral identity (2.5) can be easily derived. Indeed, by multiplying the the equation (3.1) by an arbitrary test function $\vec{\psi}(x, t)$ and integrating over $Q_{T}$, we have after the integration by parts that

$$
\iint_{Q_{T}}\left[-\vec{H}_{\varepsilon} \cdot \vec{\psi}_{t}+k\left(u_{\varepsilon}\right) \nabla \times \vec{H}_{\varepsilon} \cdot \nabla \times \vec{\psi}\right] d x d t=\int_{\Omega} \vec{H}_{0 \varepsilon} \cdot \vec{\psi}(x, 0) d x,
$$

which concludes the equation (2.5) as $\varepsilon \rightarrow 0$.

In order to show that $u(x, t)$ is a distribution solution, noting that for any vector fields $\vec{G}$ and $\vec{F}$, we have

$$
\nabla \cdot[\vec{G} \times \vec{F}]=(\nabla \times \vec{G}) \cdot \vec{F}-\vec{F} \cdot(\nabla \times \vec{G}) .
$$

we use the above formula to rewrite the right-hand term in (3.2) in the following form

$$
k\left(u_{\varepsilon}\right)\left|\nabla \times H_{\varepsilon}\right|^{2}=\nabla \cdot\left[k\left(u_{\varepsilon}\right) \vec{H}_{\varepsilon} \times\left(\nabla \times \vec{H}_{\varepsilon}\right)\right]-\vec{H}_{\varepsilon} \cdot \vec{H}_{\varepsilon t} .
$$

Let $\phi(x, t) \in C_{0}^{\infty}\left(Q_{T}\right)$. We multiply the equation (3.2) by $\phi(x, t)$ to obtain

$$
\iint_{Q_{T}}\left[\left(u_{\varepsilon}+\frac{1}{2}\left|\vec{H}_{\varepsilon}\right|^{2}\right) \phi_{t}+u \Delta \phi\right] d x d t=\iint_{Q_{T}}\left[k\left(u_{\varepsilon}\right) \vec{H}_{\varepsilon} \times\left(\nabla \times \vec{H}_{\varepsilon}\right)\right] \cdot \nabla \phi d x d t .
$$

As $u_{\varepsilon}(x, t)$ and $\vec{H}_{\varepsilon}(x, t)$ converge to $u(x, t)$ and $\vec{H}(x, t)$ almost everywhere in $Q_{T}$, we can take the limit under the integral and obtain

$$
\iint_{Q_{T}}\left[u \phi_{t}+u \triangle \phi\right] d x d t=\iint_{Q_{T}}\left[-\frac{1}{2}|\vec{H}|^{2} \phi_{t}+(k(u) \vec{H} \times(\nabla \times \vec{H})) \cdot \nabla \phi\right] d x d t .
$$

To obtain the integral identity (2.6), we choose $\vec{\psi}(x, t)=\vec{H}(x, t) \phi(x, t)$ in the identity (2.5) to see

$$
\begin{aligned}
& \iint_{Q_{T}} \frac{1}{2}|\vec{H}|^{2} \phi_{t} d x d t \\
& =\iint_{Q_{T}} k(u)|\nabla \times \vec{H}|^{2} d x d t+\iint_{Q_{T}} k(u)(\nabla \times \vec{H}) \cdot(\nabla \phi \times \vec{H}) d x d t \\
& =\iint_{Q_{T}} k(u)|\nabla \times \vec{H}|^{2} d x d t+\iint_{Q_{T}}[k(u) \vec{H} \times(\nabla \times \vec{H})] \cdot \nabla \phi d x d t,
\end{aligned}
$$


where the second equality comes from the following identity:

$$
\vec{A} \cdot[\vec{B} \times \vec{C}]=\vec{B} \cdot[\vec{C} \times \vec{A}]
$$

for any vector fields $\vec{A}, \vec{B}$ and $\vec{C}$. Consequently, we have the desired identity (2.6). This completes our proof of the theorem.

Remark 3.1: For the steady-state system, the similar existence theorem can be proved.

Remark 3.2: The uniqueness is an open question. It would also be interesting to study the regularity of solutions.

Acknowledgement: The author would like to thank Professor C. Sulem for her many helpful comments.

\section{References}

1. Xinfu Chen and A. Friedman, The thermister problem for conductivity which vanishes at large temperature, IMA preprint series No. 792.

2. G. Cimatti and G. Prodi, Existence results for a nonlinear elliptic systems modelling a temperature dependent electrical resistor, Ann. Mat. Pure ed Appl., 152(1988), 227-236.

3. D. Gilbarg and N. Trudinger, Elliptic Partial Differential Equations of Second Order, Springer-Verlag, Berlin, 2nd ed., 1983.

4. L. D. Landau and E.M. Lifshitz, Electrodynamics of Continuous Media, Pergamon Press, New York, 1960. 
5. J. Simon, Compact sets in the space $L^{p}(0, T ; B)$, Ann. Mat. Pure ed Appl., 146(1987), 65-96.

6. H. Xie and W. Allegretto, $C^{\alpha}(\Omega)$ solutions of a class of nonlinear degenerate elliptic systems arising in the thermistor problem, preprint.

7. J. M. Young, Steady state Joule heating with temperature dependent conductivities, Applied Sci. Research, 43(1986), 55-65. 
Avner Friedman and Bei Hu, The Stefan problem with kinetic condition at the free boundary

M.A. Grinfeld, The stress driven instabilities in crystals: mathematical models and physical manifestations

Bei Hu and Lihe Wang, A free boundary problem arising in electrophotography: solutions with connected toner region

Yongzhi Xu, T. Craig Poling, and Trent Brundage, Direct and inverse scattering of time harmonic acoustic waves in an inhomogeneous shallow ocean

Steven J. Altschuler, Singularities of the curve shrinking flow for space curves

Steven J. Altschuler and Matthew A. Grayson, Shortening space curves and flow through singularities Tong Li, On the Riemann problem of a combustion model

L.A. Peletier \& W.C. Troy, Self-similar solutions for diffusion in semiconductors

C.J. van Duijn, L.A. Peletier \& R.J. Schotting, On the analysis of brine transport in porous media

Minkyu Kwak, Finite dimensional description of convective reaction-diffusion equations

Minkyu Kwak, Finite dimensional inertial forms for the 2D Navier-Stokes equations

Victor A. Galaktionov and Sergey A. Posashkov, On some monotonicity in time properties for a quasilinear parabolic equation with source

Victor A. Galaktionov, Remark on the fast diffusion equation in a ball

Hi Jun Choe and Lihe Wang, A regularity theory for degenerate vector valued variational inequalities

Vladimir I. Oliker and Nina N. Uraltseva, Evolution of nonparametric surfaces with speed depending on curvature, II. The mean curvature case.

S. Kamin and W. Liu, Large time behavior of a nonlinear diffusion equation with a source

Shoshana Kamin and Juan Luis Vazquez, Singular solutions of some nonlinear parabolic equations

Bernhard Kawohl and Robert Kersner, On degenerate diffusion with very strong absorption

Avner Friedman and Fernando Reitich, Parameter identification in reaction-diffusion models

E.G. Kalnins, H.L. Manocha and Willard Miller, Jr., Models of $q$-algebra representations I. Tensor products of special unitary and oscillator algebras

Robert J. Sacker and George R. Sell, Dichotomies for linear evolutionary equations in Banach spaces

Oscar P. Bruno and Fernando Reitich, Numerical solution of diffraction problems: a method of variation of boundaries

Oscar P. Bruno and Fernando Reitich, Solution of a boundary value problem for Helmholtz equation via variation of the boundary into the complex domain

Victor A. Galaktionov and Juan L. Vazquez, Asymptotic behaviour for an equation of superslow diffusion. The Cauchy problem

Josephus Hulshof and Juan Luis Vazquez, The Dipole solution for the porous medium equation in several space dimensions

Shoshana Kamin and Juan Luis Vazquez, The propagation of turbulent bursts for a diffusion-convection equation

Marco Biroli and Umberto Mosco, Discontinuous media and Dirichlet forms of diffusion type

Stathis Filippas and Jong-Shenq Guo, Quenching profiles for one-dimensional semilinear heat equations

H. Scott Dumas, A Nekhoroshev-like theory of classical particle channeling in perfect crystals

R. Natalini and A. Tesei, On a class of perturbed conservation laws

Paul K. Newton and Shinya Watanabe, The geometry of nonlinear Schrödinger standing waves

S.S. Sritharan, On the nonsmooth verification technique for the dynamic programming of viscous flow

Mario Taboada and Yuncheng You, Global attractor, inertial manifolds and stabilization of nonlinear damped beam equations

Shigeru Sakaguchi, Critical points of solutions to the obstacle problem in the plane

F. Abergel, D. Hilhorst and F. Issard-Roch, On a dissolution-growth problem with surface tension in the neighborhood of a stationary solution

Erasmus Langer, Numerical simulation of MOS transistors

Haïm Brezis and Shoshana Kamin, Sublinear elliptic equations in $\mathbb{R}^{n}$

Johannes C.C. Nitsche, Boundary value problems for variational integrals involving surface curvatures

Chao-Nien Chen, Multiple solutions for a semilinear elliptic equation on $\mathbb{R}^{N}$ with nonlinear dependence on the gradient

D. Brochet, X. Chen and D. Hilhorst, Finite dimensional exponential atttractor for the phase field model 
Robert L. Pego and Michael I. Weinstein, A class of eigenvalue problems, with applications to instability of solitary waves

Mahmoud Affouf, Numerical study of a singular system of conservation laws arising in enhanced oil reservoirs

Darin Beigie, Anthony Leonard and Stephen Wiggins, The dynamics associated with the chaotic

866

867

868

869

870

871

872

873

874

875

876

877

878

879

880

881

882

883

884

885

886

887

888

889

890

891

892

893

894

895

896

897

898

899

900

901

902

903

904

905

906

907

908

909

910

911 of tangles two dimensional quasiperiodic vector fields: theory and applications
Gui-Qiang Chen and Tai-Ping Liu, Zero relaxation and dissipation limits for hyperbolic conservation laws

Gui-Qiang Chen and Jian-Guo Liu, Convergence of second-order schemes for isentropic gas dynamics

Aleksander M. Simon and Zbigniew J. Grzywna, On the Larché-Cahn theory for stress-induced diffusion

Jerzy Luczka, Adam Gadomski and Zbigniew J. Grzywna, Growth driven by diffusion

Mitchell Luskin and Tsorng-Whay Pan, Nonplanar shear flows for nonaligning nematic liquid crystals

Mahmoud Affouf, Unique global solutions of initial-boundary value problems for thermodynamic phase transitions

Richard A. Brualdi, Keith L. Chavey and Bryan L. Shader, Rectangular $L$-matrices

Xinfu Chen, Avner Friedman and Bei Hu, The thermistor problem with zero-one conductivity II

Raoul LePage, Controlling a diffusion toward a large goal and the Kelly principle

Raoul LePage, Controlling for optimum growth with time dependent returns

Marc Hallin and Madan L. Puri, Rank tests for time series analysis a survey

V.A. Solonnikov, Solvability of an evolution problem of thermocapillary convection in an infinite time interval

Horia I. Ene and Bogdan Vernescu, Viscosity dependent behaviour of viscoelastic porous media

Kaushik Bhattacharya, Self-accommodation in martensite

D. Lewis, T. Ratiu, J.C. Simo and J.E. Marsden, The heavy top: a geometric treatment

Leonid V. Kalachev, Some applications of asymptotic methods in semiconductor device modeling

David C. Dobson, Phase reconstruction via nonlinear least-squares

Patricio Aviles and Yoshikazu Giga, Minimal currents, geodesics and relaxation of variational integrals on mappings of bounded variation

Patricio Aviles and Yoshikazu Giga, Partial regularity of least gradient mappings

Charles R. Johnson and Michael Lundquist, Operator matrices with chordal inverse patterns

B.J. Bayly, Infinitely conducting dynamos and other horrible eigenproblems

Charles M. Elliott and Stefan Luckhaus, 'A generalised diffusion equation for phase separation of a multi-component mixture with interfacial free energy'

Christian Schmeiser and Andreas Unterreiter, The derivation of analytic device models by asymptotic methods

LeRoy B. Beasley and Norman J. Pullman, Linear operators that strongly preserve the index of imprimitivity

Jerry Donato, The Boltzmann equation with lie and cartan

Thomas R. Hoffend Jr., Peter Smereka and Roger J. Anderson, A method for resolving the laser induced local heating of moving magneto-optical recording media

E.G. Kalnins, Willard Miller, Jr. and Sanchita Mukherjee, Models of $q$-algebra representations: the group of plane motions

T.R. Hoffend Jr. and R.K. Kaul, Relativistic theory of superpotentials for a nonhomogeneous, spatially isotropic medium

Reinhold von Schwerin, Two metal deposition on a microdisk electrode

Vladimir I. Oliker and Nina N. Uraltseva, Evolution of nonparametric surfaces with speed depending on curvature, III. Some remarks on mean curvature and anisotropic flows

Wayne Barrett, Charles R. Johnson, Raphael Loewy and Tamir Shalom, Rank incrementation via diagonal perturbations

Thierry Dombre, Alain Pumir, and Eric D. Siggia, On the interface dynamics for convection in porous media Mingxiang Chen, Xu-Yan Chen and Jack K. Hale, Structural stability for time-periodic one-dimensional parabolic equations

Hong-Ming Yin, Global solutions of Maxwell's equations in an electromagnetic field with the temperaturedependent electrical conductivity

Robert Grone, Russell Merris and William Watkins, Laplacian unimodular equivalence of graphs

Miroslav Fiedler, Structure-ranks of matrices

Miroslav Fiedler, An estimate for the nonstochastic eigenvalues of doubly stochastic matrices

Miroslav Fiedler, Remarks on eigenvalues of Hankel matrices

Charles R. Johnson, D.D. Olesky, Michael Tsatsomeros and P. van den Driessche, Spectra with positive elementary symmetric functions

Pierre-Alain Gremaud, Thermal contraction as a free boundary problem

K.L. Cooke, Janos Turi and Gregg Turner, Stabilization of hybrid systems in the presence of feedback delays

Robert P. Gilbert and Yongzhi Xu, A numerical transmutation approach for underwater sound propagation

LeRoy B. Beasley, Richard A. Brualdi and Bryan L. Shader, Combinatorial orthogonality

Richard A. Brualdi and Bryan L. Shader, Strong hall matrices

Håkan Wennerström and David M. Anderson, Difference versus Gaussian curvature energies; monolayer versuss bilayer curvature energies applications to vesicle stability

Shmuel Friedland, Eigenvalues of almost skew symmetric matrices and tournament matrices 